\title{
Las habilidades blandas en estudiantes de ingeniería de tres instituciones públicas de educación superior
}

\author{
Soft Skills in Engineering Students from Three Public Higher Education \\ Institutions
}

\section{Soft skills em estudantes de engenharia de três instituições públicas de ensino superior}

\author{
Claudia Alejandra Hernández Herrera \\ Instituto Politécnico Nacional, México \\ al9505@gmail.com \\ https://orcid.org/0000-0002-4060-2941 \\ Juan Carlos Neri Torres \\ Instituto Politécnico Nacional, México \\ neritor@hotmail.com \\ https://orcid.org/0000-0002-8352-3944
}

\section{Resumen}

El objetivo del presente artículo fue analizar la percepción que tienen los estudiantes de ingeniería en relación con la adquisición de habilidades blandas obtenidas en el transcurso de sus carreras. La obtención de los datos se realizó por medio de la construcción de un cuestionario con escala tipo Likert. Se utilizó el análisis factorial y las pruebas estadísticas de Kruskal-Wallis y los modelos lineales generalizados. Es un estudio transversal; la muestra fue de 3560 jóvenes de tres universidades públicas: dos ubicadas en la Ciudad de México y una en el País Vasco, España. Como parte de los resultados, los estudiantes de la Escuela de Ingeniería de Bilbao, en el País Vasco, obtuvieron las mayores puntuaciones en relación con las habilidades blandas; le siguieron las universidades mexicanas. Además, se halló que tanto en el Instituto Politécnico Nacional (IPN) como en el Tecnológico Nacional de México (TecNM) se 


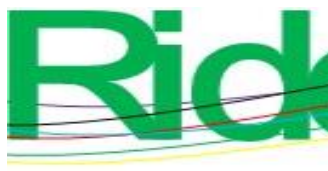

Revista Iberoamericana para la Investigación y el Desarrollo Educativo ISSN $2007-7467$

deben reforzar los saberes relacionados con este tipo de habilidades, particularmente en las ingenierías en informática y de tecnologías de la información y comunicación. Por último, los jóvenes del IPN que se encuentran trabajando son los que registraron más puntos en las también llamadas soft skills. Se concluye que las instituciones de educación tienen que trabajar en la implementación de talleres que refuercen las habilidades blandas, así como mantener el acercamiento con el sector empresarial que continúe impulsando las estancias, ya que estas coadyuvan al fortalecimiento de las competencias interpersonales de los estudiantes.

Palabras clave: carreras de ingeniería, habilidades blandas, habilidades de un ingeniero, universidades.

\section{Abstract}

The objective of this paper was to analyze the perception that engineering students have regarding the acquisition of soft skills achieved in the course of their studies. The data was obtained through of the construction of a questionnaire with a Likert-type scale; factor analysis, Kruskal-Wallis statistical tests and generalized linear models were applied. It is a cross-sectional study; the sample was 3560 young people of three public universities: two of them located in Mexico City, and one in the Euzkadi Country, Spain. It was found that the students of the Escuela de Ingeniería de Bilbao, in the Basque Country, obtained higher scores in relation to soft skills; Mexican universities were behind. In addition, it was found that in both the Instituto Politécnico Nacional (IPN) and the Tecnológico Nacional de México (TecNM) the knowledge related to soft skills must be strengthened in computer engineering and in information and communication technologies studies. Finally, young people at IPN who are also working gave higher scores to soft skills. It is concluded that educational institutions have to work in the implementation of workshops that reinforce soft skills and maintain closeness with the business sector to allow for boosting stays, since these contribute to strengthening the interpersonal skills of students.

Keywords: engineering careers, soft skills, skills of an engineer, universities. 


\section{Resumo}

O objetivo deste artigo foi analisar a percepção que os estudantes de engenharia têm sobre a aquisição de habilidades sociais obtidas durante a carreira. Os dados foram obtidos por meio da construção de um questionário em escala do tipo Likert. Foram utilizados análise fatorial, testes estatísticos de Kruskal-Wallis e modelos lineares generalizados. É um estudo transversal; A amostra foi de 3.560 jovens de três universidades públicas: duas localizadas na Cidade do México e uma no País Basco, Espanha. Como parte dos resultados, os alunos da Escola de Engenharia de Bilbao, no País Basco, obtiveram as pontuações mais altas em relação às habilidades sociais; Universidades mexicanas seguiram. Além disso, verificou-se que o Instituto Politécnico Nacional (IPN) e o Instituto Tecnológico Nacional do México (TecNM) devem reforçar o conhecimento relacionado a esse tipo de habilidades, particularmente em engenharia da computação e tecnologia da informação e comunicação . Finalmente, os jovens do IPN que estão trabalhando são os que registraram mais pontos nas chamadas habilidades sociais. Conclui-se que as instituições de ensino devem trabalhar na implementação de oficinas que reforcem as habilidades sociais, bem como manter a abordagem junto ao setor empresarial que continua promovendo as estadias, uma vez que estas contribuem para o fortalecimento das habilidades interpessoais dos alunos.

Palavras-chave: carreiras de engenharia, soft skills, habilidades de um engenheiro, universidades.

Fecha Recepción: Noviembre 2019

Fecha Aceptación: Marzo 2020

\section{Introducción}

Los países que se preocupan por brindar oportunidades para el desarrollo de competencias que son consideradas importantes para los sectores de la sociedad permiten crear las condiciones para el incremento de la productividad y el desarrollo económico de sus naciones, ya que reconocen que el contar con talento humano capaz de insertarse y crecer profesionalmente en el mercado de trabajo coadyuva a que las organizaciones logren progreso y bienestar para los individuos que en ellas laboran. Por lo anterior, la adquisición de competencias es uno de los pilares fundamentales para el crecimiento de los países y el bienestar de los ciudadanos. 


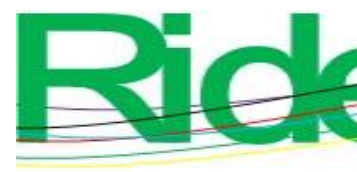

Revista Iberoamericana para la Investigación y el Desarrollo Educativo ISSN $2007-7467$

En esa línea, de acuerdo con Ibarraran, Ripani, Taboada, Villa y Garcia (2014), existe evidencia de que las habilidades blandas incrementan la empleabilidad. Y por supuesto, permiten mejorar el desempeño laboral.

En el mercado de trabajo los empleadores buscan personas que sean confiables, creativas, con una comunicación efectiva, con iniciativa, que sean disciplinadas y que demuestren actitud positiva (Majid, Liming, Tong y Raihana, 2012). Además, el sector laboral a menudo se queja de que los egresados de la educación superior no cuentan con las habilidades para el desarrollo de ciertos puestos de trabajo (Taylor, 2016).

Por su parte, las instituciones de educación superior tienen la responsabilidad de asegurar que los jóvenes estén adquiriendo las competencias necesarias para el mercado laboral. De hecho, la empleabilidad de los egresados se ha convertido en un indicador de calidad para muchas universidades (Lennox y Roos, 2017). De acuerdo con Retnanto, Parsaei y Parsaei (2019), las escuelas que imparten programas de ingeniería requieren fortalecer las habilidades sociales en los estudiantes. No obstante, otro de los problemas que enfrentan las universidades y sus profesores es que no cuentan con una forma clara de evaluar las competencias blandas.

Particularmente, en las escuelas de ingeniería los estudiantes pasan mayor tiempo perfeccionando sus habilidades técnicas y en ocasiones dejan de lado las competencias que les permiten resolver problemas de otra índole. Ante ello es necesario conocer las formas que más aprecian y las que generan mayor entusiasmo en los alumnos a la hora de aprender (Kumar y Hsiao, 2007). Las habilidades sociales son cruciales para los ingenieros, ya que el entorno laboral en el que se desarrollan cada vez es más interdisciplinario y demanda mayor trabajo colaborativo (Berglund y Heintz, 2014).

Al respecto, Florea y Stray (2018) analizaron anuncios de empleos en 33 países y encontraron que las compañías desean contratar ingenieros capaces de comunicarse, posean habilidades analíticas y resuelvan problemas. También hallaron que existen nuevas categorías de habilidades blandas como la ética en el trabajo, enfoque en el cliente y saber trabajar bajo presión.

El objetivo del presente artículo es analizar la percepción que tienen los estudiantes de ingeniería en tres universidades públicas, dos ubicadas en la Ciudad de México y una en el País Vasco, en España, en relación con las habilidades blandas que han logrado adquirir durante su transitar en la carrera. Lo anterior para reconocer los escenarios sobre los cuales cada una de las escuelas se encuentra con respecto al 


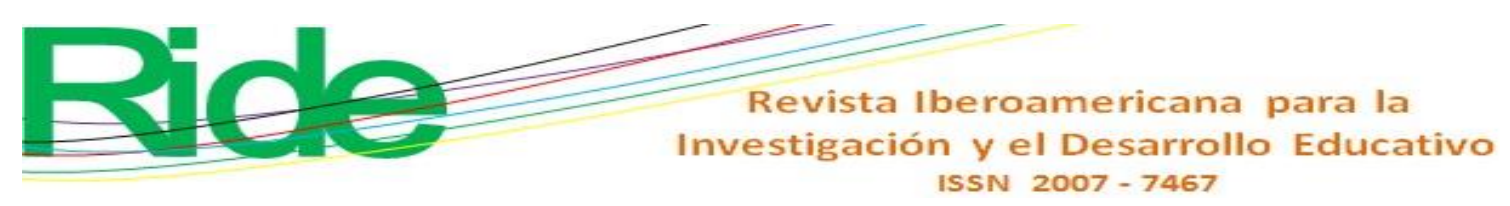

fenómeno ya especificado, y de esa forma identificar áreas de oportunidad para los futuros ingenieros.

La investigación se llevó a cabo en tres instituciones de educación superior, dos mexicanas y una española, como ya se mencionó. En lo que respecta a las mexicanas, se decidió trabajar con el Instituto Politécnico Nacional (IPN) y el Tecnológico Nacional de México (TecNM) porque ambas son reconocidas a nivel nacional por impartir carreras en ingeniería y por su ubicación geográfica; las dos acordaron participar en el estudio. En el caso de la escuela en España, se trabajó con la Universidad del País Vasco, considerada entre las 500 mejores universidades del mundo, y en específico con la Escuela de Ingeniería de Bilbao, la cual estuvo de acuerdo en colaborar.

\section{Las habilidades blandas}

Alinear la educación superior a las necesidades del mercado laboral permite incrementar las capacidades que determinan el éxito estudiantil y la empleabilidad (Cleary, Kerrigan y Van Noy, 2017). Aquí se concuerda con Fadel y Groff (2019) cuando mencionan que las escuelas tienen que impulsar una educación que apoye a forjar el carácter de los jóvenes para que adquieran y fortalezcan virtudes, valores y una capacidad de tomar decisiones para llevar una vida equilibrada, incluso se debe enseñar lo relacionado con la salud mental, la curiosidad, la resiliencia, la ética y el liderazgo.

Las habilidades blandas y sociales tienen un gran valor para los jóvenes ya que están relacionadas con el desempeño laboral y el desarrollo profesional. Además, las competencias interpersonales ayudan a aquellas personas cuyo trabajo demanda manejar de forma adecuada el aspecto emocional para mantener una relación sana con los clientes. Aunado a ello, proporcionan habilidades de gestión y liderazgo para apoyar a los equipos de trabajo para que alcancen sus objetivos.

También conocidas como competencias transversales, aluden a rasgos personales que coadyuvan a incrementar la positividad y mejorar las relaciones entre las personas. Asimismo, estas competencias permiten saber qué tanto se conocen las personas y su capacidad de manejarse a sí mismas. Lo anterior está relacionado con el desarrollo personal que involucra componentes emocionales, conductuales y cognitivos, sin dejar de lado la inteligencia emocional y la social (Dell'Aquila et al., 2017). 


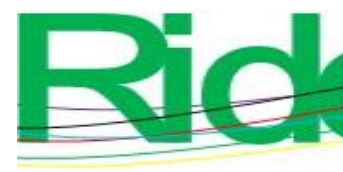

Revista Iberoamericana para la

Investigación y el Desarrollo Educativo

ISSN 2007 - 7467

Siguiendo a Dell'Aquila et al. (2017) y Lennox y Roos (2017), las competencias blandas o inteligencias interpersonales son aquellas que permiten la creación y desarrollo de relaciones positivas con los demás, además de ser competentes clave en la resolución positiva de conflictos. Como ya se adelantaba líneas arriba, la inteligencia intrapersonal está relacionada con la comprensión que los sujetos tienen de sí mismos: quien tiene esta inteligencia desarrollada es más proclive a conocer y reconocer sentimientos personales, temores y motivaciones. Lo anterior tiene que ver con la introspección y la autorreflexión que coadyuva a la autoconciencia.

Por su parte, Pieterse y Van Eekelen (2016) señalan que es posible que las habilidades interpersonales sean un elemento importante para obtener un empleo, así como que este tipo de competencias permiten convencer a las personas de que pueden ser capaces de progresar y tener éxito en la organización. En el mundo de las organizaciones se valoran las habilidades de la comunicación, la gestión, la planificación, el trabajo en equipo, la colaboración y las relaciones interpersonales.

Robles (2012) enlista las 10 habilidades blandas más relevantes en el contexto laboral: integridad, comunicación, cortesía, responsabilidad, habilidades sociales, actitud positiva, profesionalismo, flexibilidad, trabajo en equipo y ética laboral. Las habilidades blandas tienen que ver más con las personas que con los conocimientos que poseen. Por estas razones y otras más, las habilidades blandas deben de considerarse como una inversión.

Este conjunto de habilidades incluye a todas aquellas que son valiosas en los ambientes de trabajo, sustentan el comportamiento de los individuos en su empleo, aunque, por lo mismo, son difíciles de medir. Cabe especificar que no se deben confundir con valores, creencias, rasgos y conductas (Matteson, Anderson y Boyden, 2016).

Es igualmente importante recalcar que las habilidades blandas apoyan a los jóvenes a fortalecer su confianza para que puedan desenvolverse de mejor manera en los entornos de trabajo. Además, cuando los jóvenes tienen la oportunidad de obtener experiencias en el campo laboral y aplicar las competencias blandas, adquieren mayores perspectivas de su profesión. Este tipo de habilidades permite a las personas hacerles frente a los desafíos de la vida. También son predictores del éxito en la vida, pero esto depende de los rasgos de personalidad, tal y como la conciencia, la perseverancia, la sociabilidad y la curiosidad. Abdullah-Al-Mamun (2012) dice que dichis rasgos no están relacionados con la inteligencia. 


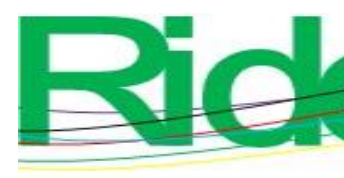

Revista Iberoamericana para la Investigación y el Desarrollo Educativo ISSN $2007-7467$

Según Rao (2014), las habilidades blandas son conocidas como habilidades para la vida, habilidades interpersonales, habilidades de empleabilidad y de inteligencia emocional. Se trata de habilidades que contribuyen al éxito en la vida y en el mundo laboral. Y se pueden aprender en un entorno de entrenamiento. No obstante, cuando se carece de apoyo, las habilidades que ya se adquirieron pueden perderse (Gibb, 2014). Al respecto, Taylor (2016) subraya que estas son valiosas pero que son difíciles de enseñar. Complementa que se encuentran relacionadas con la inteligencia emocional, la actitud positiva, la disposición por aprender y la autogestión del tiempo.

Bailly y Léné (2012) señalan algo interesante: las habilidades blandas son aquellas que tienen que ver con la personalidad del empleado como el entusiasmo, la empatía con los clientes y la capacidad de tener interacción social, y son consideradas innatas a los individuos; sin embargo, existe la percepción de que se trata de una nueva forma de discriminación al momento de emplear. Mientras que Balcar (2014) comenta que las habilidades blandas son competencias intangibles que son complejas de medir y están vinculadas con las actitudes del individuo. Sea como fuere, son competencias que tienen una relación positiva con los niveles salariales de las personas.

Por último, Rao (2014) apuntan que las competencias blandas se apoyan de las habilidades duras. Las combinaciones de saberes permiten desarrollar líderes capaces de influir en las personas; líderes que manejan y gestionan la incertidumbre; saben usar de forma adecuada el tiempo y delegan con facilidad; conocen las formas de alinear esfuerzos; son capaces, en suma, de aprender y crecer de forma continua y alcanzan objetivos organizacionales.

\section{Materiales y métodos}

El estudio aquí en cuestión es cuantitativo y transversal. En la investigación se trabajó con tres instituciones de educación superior públicas, dos de ellas ubicadas en la Ciudad de México y otra en España. Al momento de la selección de las escuelas influyó el hecho de que contaran con carreras de ingeniería y que mostraran disposición para participar en el estudio. En el caso mexicano, participó, en primer lugar, el IPN, a través de la Unidad Profesional Interdisciplinaria de Ingeniería y Ciencias Sociales y Administrativas (Upiicsa); las carreras que representaron a esta institución fueron ingeniería en Transporte, ingeniería Industrial e ingeniería en Informática. La segunda institución fue el TecNM, mediante el Instituto Tecnológico de Gustavo A. Madero, a 


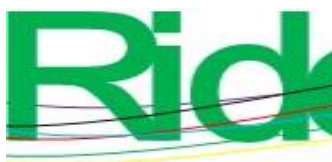

Revista Iberoamericana para la Investigación y el Desarrollo Educativo ISSN 2007 - 7467

través de las ingenierías en Tecnologías de la Información y Comunicaciones, en Gestión Empresarial, en Logística, Industrial y la ingeniería Ambiental. Finalmente, de la Escuela de Ingeniería de Bilbao, ubicada en el País Vasco, los grados participantes fueron ingeniería Ambiental, ingeniería en Organización Industrial, ingeniería en Tecnología Industrial, ingeniería Eléctrica, ingeniería Electrónica Industrial y Automática, ingeniería Mecánica, ingeniería en Tecnología de Telecomunicación, ingeniería Civil y la ingeniería de Tecnología de Minas y Energía. Las edades de los sujetos oscilaban entre los 18 y 24 años; la edad promedio fue de 21.66 años.

El muestreo que se empleó fue por conveniencia: por la proximidad y facilidad de los sujetos. En total fueron 3560 cuestionarios: 2130 son jóvenes del IPN, 500 de la Universidad del País Vasco y 930 del TecNM. Los datos se recabaron por medio de la construcción de un cuestionario que cuenta con 18 enunciados, los cuales miden la percepción respecto a la adquisición de habilidades blandas. Asimismo, se incluyó un listado de 25 competencias y se les pidió a los participantes que eligieran cinco de estas que consideraran relevantes en la formación de los ingenieros. Se empleó una escala tipo Likert, en donde $1=$ Nunca, $2=$ Casi siempre, $3=$ A veces, $4=$ Casi siempre y $5=$ Siempre. El instrumento fue piloteado en una de las unidades de IPN, con una muestra de 60 jóvenes. Los datos fueron procesados con el software Statistical Packet for the Social Sciences (SPSS-IBM). La confiabilidad del instrumento arrojó un alfa de Cronbach de 0.863, lo que indica que los enunciados demuestran consistencia interna. Aunado a ello, se llevó a cabo un análisis de factores exploratorio. Las pruebas estadísticas arrojaron un Kaiser-Meyer-Olkin de $0.940>0.50$, lo que significa que los ítems tienen la capacidad de agruparse en dimensiones; se trabajó únicamente con un factor que integra las habilidades blandas. Es importante mencionar que se tomó la decisión de hacer uso del método de análisis factorial pues permite estudiar grandes cantidades de datos. La extracción fue por medio de los componentes principales y la rotación fue varimax (ver tabla 1). Para la aplicación de los instrumentos, en el caso de México, se entrenaron a jóvenes ayudantes de investigación; en España, los cuestionarios contestados fueron producto de una estancia de investigación. Durante la aplicación del cuestionario se enfatizó en que se resguardaría la confidencialidad de los informantes.

La primera parte del cuestionario incluye las variables contextuales: el género, la edad, la escuela, la carrera, el promedio, el turno, las materias reprobadas, las formas de aprender, la percepción de cuánto gana un egresado, el semestre, la razón de la asistencia, 
si actualmente cuenta con trabajo, si cuentan con beca, el idioma, la escolaridad y el oficio del padre y la escolaridad y el oficio de la madre.

Tabla 1. Análisis factorial, componentes rotados

\begin{tabular}{|c|c|c|c|c|}
\hline $\begin{array}{l}\text { Variable. Factor uno, } \\
\text { habilidades blandas }\end{array}$ & $\begin{array}{l}\text { Coeficiente } \\
\text { de } \\
\text { correlación }\end{array}$ & $\begin{array}{c}\text { IPN. } \\
\text { Promedio } \\
\text { por ítem }\end{array}$ & $\begin{array}{c}\text { Escuela de } \\
\text { Ingeniería } \\
\text { de Bilbao. } \\
\text { Promedio } \\
\text { por ítem }\end{array}$ & $\begin{array}{c}\text { TecNM. } \\
\text { Promedio por } \\
\text { ítem }\end{array}$ \\
\hline $\begin{array}{l}\text { Crees que te puedes } \\
\text { comunicar de manera } \\
\text { eficaz con tu entorno. }\end{array}$ & 0.631 & 3.70 & 3.85 & 3.59 \\
\hline $\begin{array}{l}\text { Consideras que eres } \\
\text { flexible al cambio de un } \\
\text { proceso o forma de } \\
\text { trabajo. }\end{array}$ & 0.604 & 3.85 & 3.72 & 3.67 \\
\hline $\begin{array}{l}\text { Consideras que procesas } \\
\text { información de manera } \\
\text { rápida y eficaz. }\end{array}$ & 0.604 & 3.62 & 3.78 & 3.38 \\
\hline $\begin{array}{l}\text { Consideras contar con la } \\
\text { habilidad para resolver } \\
\text { conflictos. }\end{array}$ & 0.571 & 3.84 & 3.81 & 3.60 \\
\hline $\begin{array}{l}\text { Capacidad para trabajar } \\
\text { en equipo. }\end{array}$ & 0.557 & 3.73 & 3.65 & 3.61 \\
\hline $\begin{array}{l}\text { Piensas que afrontas los } \\
\text { retos con facilidad. }\end{array}$ & 0.556 & 3.67 & 3.75 & 3.61 \\
\hline $\begin{array}{l}\text { Capacidad para tener } \\
\text { nuevas ideas, alternativas } \\
\text { y soluciones. }\end{array}$ & 0.556 & 4.03 & 3.88 & 3.76 \\
\hline $\begin{array}{l}\text { Capacidad para separar lo } \\
\text { personal de lo laboral. }\end{array}$ & 0.523 & 3.62 & 4.09 & 3.66 \\
\hline $\begin{array}{l}\text { Retroalimentación de tus } \\
\text { errores para poder } \\
\text { corregirlos. }\end{array}$ & 0.519 & 3.79 & 3.97 & 3.60 \\
\hline $\begin{array}{l}\text { Realizas autocríticas y/o } \\
\text { autoanálisis continuos } \\
\text { para seguir mejorando } \\
\text { como persona. }\end{array}$ & 0.511 & 3.92 & 3.98 & 3.71 \\
\hline $\begin{array}{l}\text { Consideras que te } \\
\text { relacionas con facilidad. }\end{array}$ & 0.508 & 3.68 & 3.87 & 3.47 \\
\hline $\begin{array}{l}\text { Te consideras persuasivo } \\
\text { e influyente en las } \\
\text { decisiones de las demás } \\
\text { personas. }\end{array}$ & 0.507 & 3.32 & 3.51 & 3.20 \\
\hline $\begin{array}{l}\text { Consideras que cuentas } \\
\text { con iniciativa y decisión } \\
\text { en la resolución de } \\
\text { problemas. }\end{array}$ & 0.504 & 3.63 & 4.06 & 3.45 \\
\hline
\end{tabular}




\begin{tabular}{|l|l|l|l|l|}
\hline $\begin{array}{l}\text { Te mantienes informado } \\
\text { acerca de las tendencias } \\
\text { del mercado laboral. }\end{array}$ & 0.502 & 3.61 & 3.70 & 3.40 \\
\hline $\begin{array}{l}\text { Reaccionas con calma a } \\
\text { situaciones de adversidad }\end{array}$ & 0.458 & 3.32 & 3.33 & 3.31 \\
\hline $\begin{array}{l}\text { Piensas que le das a los } \\
\text { medios electrónicos el } \\
\text { máximo que } \\
\text { aprovechamiento dentro } \\
\text { de tu área de estudio y/o } \\
\text { laboral. }\end{array}$ & 0.431 & 3.56 & 3.57 & 3.46 \\
\hline $\begin{array}{l}\text { Consideras } \\
\text { cuestionas las ideas tanto } \\
\text { propias como de la gente } \\
\text { que te rodea. }\end{array}$ & 0.427 & 3.48 & 3.85 & 3.52 \\
\hline $\begin{array}{l}\text { Crees que el aprendizaje } \\
\text { obtenido te ayuda a } \\
\text { fortalecer tus actitudes de } \\
\text { liderazgo. }\end{array}$ & 0.323 & 3.66 & 2.93 & 3.64 \\
\hline
\end{tabular}

Fuente: Elaboración a partir de los datos obtenidos del software SPSS

Es un estudio transversal. El levantamiento de los datos se llevó a cabo en los meses de mayo, junio y julio del 2018. En el caso de México, se entrenaron a jóvenes que apoyaron en la aplicación del cuestionario; en el caso de España, los instrumentos fueron realizados gracias a una estancia de investigación. Los enunciados fueron construidos a partir de la literatura y permiten analizar las percepciones de los jóvenes en relación con la obtención de habilidades blandas.

Los supuestos que se establecieron fueron:

a) Existen diferencias o similitudes entre las percepciones de los alumnos en relación con las habilidades blandas obtenidas de las tres escuelas participantes.

b) Existen diferencias en relación con el factor de competencias blandas y las variables contextuales del modelo lineal generalizado integrado por las variables del género, la carrera, las asignaturas reprobadas, el aprendizaje, cuánto gana un egresado y la razón de asistencia.

c) Existe diferencias en relación con el factor de competencias blandas y las variables contextuales del modelo lineal generalizado compuesto por actividad laboral actual, becas, idiomas, escolaridad y oficio del padre, escolaridad y oficio de la madre, oficio del papá y oficio de la mamá.

Se realizó el análisis factorial para identificar la capacidad de agrupación de los enunciados. Además, ese tipo de pruebas estadísticas permiten estudiar grandes cantidades de datos. Se trabajó con la prueba de Kruskal-Wallis, prueba no paramétrica para muestras independientes para determinar la igualdad o diferencia entre los grupos. 
De igual forma, se decidió trabajar con los modelos lineales generalizados, ya que estos permiten ubicar las posibles diferencias de los grupos de variables estudiados con respecto a los factores. Es importante mencionar que los modelos lineales generalizados se dividieron en dos partes debido a la cantidad de datos que se trabajaron. Las variables que se contrastaron con el factor de habilidades blandas fueron: 1) género, 2) carrera, 3) materias reprobadas, 4) formas de aprender (visual y auditiva), 5) percepción de cuánto gana un egresado, 6) semestre, 7) razón de asistencia a la escuela, 8) cuenta actualmente con trabajo, 9) becas, 10) idiomas, 11) escolaridad de la mamá y 12) escolaridad del padre (ver tabla 5).

\section{Resultados}

Tal y como se especificó, se aplicó la prueba no paramétrica de Kruskal-Wallis para muestras independientes. Lo anterior para identificar la similitud o diferencias entre las puntuaciones del factor uno y las tres instituciones educativas que se estudiaron. De acuerdo con los resultados obtenidos de la prueba estadística, se halló la existencia de la no similitud entre las puntuaciones de cada escuela $(P>0.05)$ (ver tabla 2$)$. Ahora bien, en los rangos promedio se observa que la universidad en España es la que obtiene mayores puntos en el factor que evalúa las competencias blandas de los jóvenes, le siguen los jóvenes del IPN y, por último, los alumnos del TecNM.

Tabla 2. Prueba de Kruskal Wallis, factor habilidades blandas

\begin{tabular}{|l|l|l|}
\hline Escuela & $\boldsymbol{N}$ & $\begin{array}{l}\text { Rango } \\
\text { promedio }\end{array}$ \\
\hline IPN & 2118 & 1819.29 \\
Escuela de Ingeniería de Bilbao & 500 & $\mathbf{1 9 4 3 . 7 1}$ \\
TecNM & 924 & 1567.04 \\
\hline Ji al cuadrado & 55.827 \\
Grados de libertad & 2 & \\
$P$-value & 0.000 \\
\hline
\end{tabular}

Fuente: Elaboración a partir de los datos obtenidos del software SPSS 
Tabla 3. Resultados de las variables independientes

\begin{tabular}{|c|c|c|c|c|}
\hline \multicolumn{2}{|l|}{ Variables } & IPN & $\begin{array}{l}\text { Escuela de } \\
\text { Ingeniería } \\
\text { de Bilbao }\end{array}$ & TecNM \\
\hline \multirow[t]{2}{*}{ Género } & Masculino & $60 \%$ & $57 \%$ & $61 \%$ \\
\hline & Femenino & $40 \%$ & $43 \%$ & $39 \%$ \\
\hline \multirow[t]{17}{*}{ Carreras } & Ingeniería en Informática & $22 \%$ & - & - \\
\hline & Ingeniería en Transporte & $40 \%$ & - & - \\
\hline & Ingeniería Industrial & $38 \%$ & - & - \\
\hline & Ingeniería Civil & - & $6 \%$ & \\
\hline & Ingeniería de Minas y Energía & - & $7 \%$ & \\
\hline & Ingeniería Electrónica & - & $2 \%$ & \\
\hline & $\begin{array}{l}\text { Ingeniería en Tecnología } \\
\text { Industrial }\end{array}$ & - & $62 \%$ & \\
\hline & $\begin{array}{l}\text { Ingeniería en } \\
\text { Telecomunicaciones }\end{array}$ & - & $11 \%$ & \\
\hline & Ingeniería Mecánica & - & $4 \%$ & \\
\hline & $\begin{array}{l}\text { Ingeniería en Organización } \\
\text { Industrial }\end{array}$ & - & $3 \%$ & \\
\hline & Ingeniería Eléctrica & - & $2 \%$ & \\
\hline & Ingeniería Ambiental & - & $3 \%$ & \\
\hline & $\begin{array}{l}\text { Ingeniería en Tecnologías de } \\
\text { la Información y } \\
\text { Comunicaciones }\end{array}$ & - & - & $34 \%$ \\
\hline & $\begin{array}{l}\text { Ingeniería en Gestión } \\
\text { Empresarial }\end{array}$ & - & - & $22 \%$ \\
\hline & Ingeniería en Logística & - & - & $18 \%$ \\
\hline & Ingeniería Industrial & - & - & $17 \%$ \\
\hline & Ingeniería Ambiental & - & - & $5 \%$ \\
\hline \multirow{5}{*}{$\begin{array}{l}\text { Ingresos } \\
\text { mensuales } \\
\text { familiares }\end{array}$} & De 4000 a 6000 pesos & $9 \%$ & - & $17 \%$ \\
\hline & De 6000 a 9000 pesos & $41 \%$ & - & $45 \%$ \\
\hline & Más de 10000 pesos & $50 \%$ & - & $38 \%$ \\
\hline & De 4000 a 6000 euros & - & $78 \%$ & - \\
\hline & De 6000 a 9000 euros & - & $18 \%$ & - \\
\hline
\end{tabular}




\begin{tabular}{|c|c|c|c|c|}
\hline & Más de 10000 euros & - & $2 \%$ & - \\
\hline \multirow{2}{*}{$\begin{array}{l}\text { El } \\
\text { estudiante } \\
\text { cuenta con } \\
\text { trabajo }\end{array}$} & Sí & $32 \%$ & $11 \%$ & $37 \%$ \\
\hline & No & $68 \%$ & $89 \%$ & $63 \%$ \\
\hline \multirow{2}{*}{$\begin{array}{l}\text { El } \\
\text { estudiante } \\
\text { cuenta con } \\
\text { beca }\end{array}$} & Sí & $27 \%$ & $17 \%$ & $27 \%$ \\
\hline & No & $73 \%$ & $83 \%$ & $73 \%$ \\
\hline \multirow{6}{*}{$\begin{array}{l}\text { Escolaridad } \\
\text { del padre }\end{array}$} & No tiene estudios & $1 \%$ & $0 \%$ & $2 \%$ \\
\hline & Primaria & $7 \%$ & $0 \%$ & $7 \%$ \\
\hline & Secundaria & $21 \%$ & $13 \%$ & $28 \%$ \\
\hline & Preparatoria & $37 \%$ & $19 \%$ & $33 \%$ \\
\hline & Licenciatura & $28 \%$ & $52 \%$ & $20 \%$ \\
\hline & Posgrado & $6 \%$ & $16 \%$ & $10 \%$ \\
\hline \multirow{3}{*}{$\begin{array}{l}\text { A qué se } \\
\text { dedica el } \\
\text { padre }\end{array}$} & $\begin{array}{l}\text { Trabaja en una empresa o } \\
\text { Gobierno }\end{array}$ & $53 \%$ & $59 \%$ & $49 \%$ \\
\hline & Trabaja por su cuenta & $50 \%$ & $16 \%$ & $37 \%$ \\
\hline & Es desempleado & $7 \%$ & $25 \%$ & $14 \%$ \\
\hline \multirow{6}{*}{$\begin{array}{l}\text { Escolaridad } \\
\text { de la madre }\end{array}$} & No tiene estudios & $1 \%$ & $0 \%$ & $3 \%$ \\
\hline & Primaria & $9 \%$ & $2 \%$ & $10 \%$ \\
\hline & Secundaria & $28 \%$ & $9 \%$ & $31 \%$ \\
\hline & Preparatoria & $38 \%$ & $26 \%$ & $32 \%$ \\
\hline & Licenciatura & $20 \%$ & $45 \%$ & $17 \%$ \\
\hline & Posgrado & $4 \%$ & $18 \%$ & $7 \%$ \\
\hline \multirow{3}{*}{$\begin{array}{l}\text { A qué se } \\
\text { dedica la } \\
\text { madre }\end{array}$} & $\begin{array}{l}\text { Trabaja en una empresa o } \\
\text { Gobierno }\end{array}$ & $27 \%$ & $49 \%$ & $23 \%$ \\
\hline & Trabaja por su cuenta & $25 \%$ & $15 \%$ & $25 \%$ \\
\hline & Se dedica al hogar & $48 \%$ & $36 \%$ & $46 \%$ \\
\hline
\end{tabular}

Fuente: Elaboración propia

\section{Resultados de modelo lineal generalizado del factor de habilidades}

\section{blandas}

El modelo lineal generalizado permite identificar las variaciones relacionadas con las categorías formadas por las variables independientes. El modelo se dividió en dos partes. La primera parte analiza las variables del género, la carrera, las asignaturas reprobadas, el aprendizaje, cuánto gana un egresado y la razón de asistencia. Por medio de la prueba de contraste de ómnibus, se obtuvo que en la escuela del IPN y en el TecNM se tienen variaciones significativas (ver tabla 4). De acuerdo con las pruebas de los efectos del modelo, se encontró variación en la escuela del IPN en las variables de carrera $(P<$ 0.05), materias reprobadas $(P<0.05)$, formas de aprender $(P<0.05)$, percepción de 


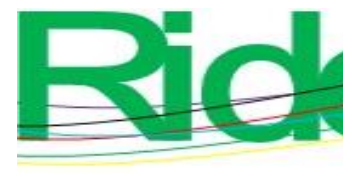

Revista Iberoamericana para la Investigación y el Desarrollo Educativo ISSN 2007 - 7467

cuánto gana un egresado $(P<0.05)$ y razón de la asistencia $(P<0.05)$ (ver tabla 5$)$. Siguiendo con el análisis de la escuela del IPN, se halló que la carrera la ingeniería Industrial es la que mejor puntuó el factor de las habilidades blandas; la ingeniería en Informática es la que obtuvo menor puntuación. Por lo que respecta a la variable de las materias reprobadas, se halló que los alumnos que reportan cero materias reprobadas son lo que califican de mejor forma el factor: los que dijeron tener más de cuatro asignaturas sin acreditar son lo que obtuvieron menor puntuación. Además, se encontró que los jóvenes que indicaron que aprenden de mejor forma cuando el profesor expone son los que alcanzaron las mejores puntuaciones en las habilidades blandas, mientras que los alumnos que señalaron que aprenden mejor cuando el profesor hace uso de imágenes son los que calificaron con menos puntuación las habilidades. Aunado a lo anterior, se encontró que, conforme los estudiantes avanzan por semestre, su percepción sobre la adquisición de las habilidades blandas es positiva. Otro hallazgo es que los jóvenes que percibieron que un egresado gana aproximadamente 10000 pesos al mes son los que obtuvieron más puntos en las competencias blandas, mientras que los que piensan que un egresado gana entre 4000 y 6000 pesos son los que obtuvieron menos puntos.

Por último, siguiendo con el caso del IPN, se encontraron diferencias con respecto a las razones de la asistencia a la escuela: los que mejor puntuaron el factor de las competencias blandas son aquellos jóvenes que indican que asisten a la universidad porque les gusta la carrera que están estudiando; los que califican con menos puntos son aquellos que señalan que estudian lo que los miembros de su familia han estudiado.

Ahora bien, en el caso del TecNM, siguiendo los efectos del modelo en su primera parte, se presentaron diferencias en las variables de la carrera $(P<0.05)$, las materias reprobadas $(P<0.05)$, las formas de aprender $(P<0.05)$ y la razón de la asistencia $(P<0.05)$. En el caso de la carrera, se halló que la que mejor evalúa las habilidades blandas es la ingeniería Industrial; la que menos califica a las competencias interpersonales es la ingeniería en Tecnologías de la Información y Comunicaciones. Asimismo, se encontró que los participantes que dijeron no tener asignaturas reprobadas son los que mejor perciben la adquisición de habilidades blandas. También que los jóvenes que califican de forma positiva los elementos de las habilidades blandas son aquellos que indican que aprenden mejor cuando el profesor hace uso de imágenes. Además, los estudiantes que perciben las habilidades blandas son aquellos que señalan que se encuentran estudiando las carreras que les gusta; los que menos califican el factor son aquellos que dicen que estudian lo que en su familia han estudiado por tradición. 
El modelo lineal generalizado del factor habilidades blandas parte dos se corrió haciendo uso de las variables independientes: 1) trabajo, 2) becas, 3) idiomas, 4) escolaridad del papá, 5) escolaridad de la mamá, 6) oficio del papá y 7) oficio de la mamá. En la prueba de contraste de ómnibus se halló que la escuela del IPN es la que tiene diferencia significativa en las variables $(P<0.05)$ (ver tabla 4$)$. En los efectos del modelo, se encontró que en el IPN las variables con diferencias son la actividad laboral, contar con una beca, el idioma y la escolaridad de la mamá (ver tabla 5). Cabe destacar que los jóvenes que indicaron que actualmente trabajan son lo que mejor califican las habilidades blandas. Además, los participantes que indicaron tener una beca son los que perciben de mejor forma el factor. Por lo que se refiere al idioma, se halló que los participantes que dijeron conocer el idioma francés calificaron de mejor forma las habilidades interpersonales. Por último, los jóvenes que señalaron que su madre no tiene estudios son lo que obtuvieron más puntos en los enunciados.

Tabla 4. Prueba de contraste de ómnibus, factor de habilidades blandas primera parte

\begin{tabular}{|c|l|l|l|}
\hline \multirow{2}{*}{$\begin{array}{c}\text { Parte del } \\
\text { modelo }\end{array}$} & Escuela & $\begin{array}{l}\text { Chi- } \\
\text { cuadrado de } \\
\text { razón de } \\
\text { verosimilitud }\end{array}$ & P-value \\
\hline \multirow{2}{*}{$\begin{array}{c}\text { Parte 1. } \\
\text { Modelo } \\
\text { lineal } \\
\text { generalizado }\end{array}$} & $\begin{array}{l}\text { Escuela de } \\
\text { Ingeniería de }\end{array}$ & 156.615 & 0.000 \\
\cline { 2 - 4 } & Bilbao & 10.175 & \\
\hline \multirow{2}{*}{$\begin{array}{c}\text { Parte 2. } \\
\text { Modelo } \\
\text { lineal } \\
\text { generalizado }\end{array}$} & $\begin{array}{l}\text { Escuela de } \\
\text { Ingeniería de }\end{array}$ & & \\
\cline { 2 - 4 } & Bilbao & 107.327 & 0.000 \\
\cline { 2 - 4 } & TecNM & 62.163 & 0.000 \\
\hline
\end{tabular}

Fuente: Elaboración propia 
Tabla 5. Modelo lineal generalizado de la percepción de las habilidades blandas en las tres universidades

\begin{tabular}{|c|c|c|c|}
\hline Variables & IPN & $\begin{array}{l}\text { Escuela } \\
\text { de } \\
\text { Ingeniería } \\
\text { de Bilbao }\end{array}$ & TecNM \\
\hline Género & - & - & - \\
\hline Carrera & $* *$ & - & $* *$ \\
\hline Materias reprobadas & $* *$ & - & $*$ \\
\hline Aprendizaje & $* *$ & - & $* *$ \\
\hline $\begin{array}{l}\text { Cuánto gana un } \\
\text { egresado }\end{array}$ & $* *$ & - & - \\
\hline Semestre & $* *$ & - & - \\
\hline Razón de la asistencia & $* *$ & - & $* *$ \\
\hline Trabajo & $*$ & - & - \\
\hline Beca & $*$ & - & - \\
\hline Idioma & $*$ & - & $*$ \\
\hline Escolaridad del padre & - & - & - \\
\hline Escolaridad de la madre & $*$ & - & - \\
\hline Oficio del padre & - & $*$ & - \\
\hline Oficio de la madre & - & - & - \\
\hline
\end{tabular}

Nota: * significa que $P<0.05 \mathrm{y} * *$ significa que $P<0.01 \mathrm{y}$ - indica que no se encontró diferencia $(P>0.05)$.

Fuente: Elaboración propia

Como parte de la encuesta, se les pidió a los estudiantes que marcaran las cinco competencias, de una lista de 26 , que ellos consideran que son de mayor importancia para un ingeniero a la hora de ejercer su profesión. En la tabla 6 se muestran las cinco competencias más importantes por cada institución. 
Tabla 6. Resultados de las cinco competencias que debe tener un ingeniero

\begin{tabular}{|l|l|l|l|l|l|}
\hline IPN & Media & $\begin{array}{l}\text { Escuela de } \\
\text { Ingeniería de Bilbao }\end{array}$ & Media & TecNM & Medi \\
\hline Liderazgo & 1.42 & Resolución de & 1.30 & Comunicación & 1.53 \\
Comunicación & 1.52 & problemas & 1.40 & Innovación & 1.65 \\
Toma de & 1.54 & Trabajo en equipo & 1.48 & Trabajo en equipo & 1.66 \\
decisiones & 1.57 & $\begin{array}{l}\text { Toma de decisiones } \\
\text { Innovación }\end{array}$ & 1.55 & Liderazgo & 1.68 \\
Resolución de & 1.58 & Liderazgo & 1.67 & Uso de las TIC & 1.68 \\
problemas & & & & & \\
\hline
\end{tabular}

Fuente: Elaboración propia

\section{Discusión}

Los hallazgos encontrados reconocen que los estudiantes de la universidad en España, a diferencia de los alumnos mexicanos, son los que perciben de forma más positiva las competencias blandas hasta ahora adquiridas. Los jóvenes españoles son lo que mejor califican la competencia de la comunicación, el procesamiento de información, son capaces de afrontar retos con facilidad, tienen la habilidad de trabajar en equipo, poseen iniciativa y decisión para resolver problemas, retroalimentan de forma correcta los errores, se relacionan con facilidad, son persuasivos e influyentes en las decisiones que toman las demás personas, llevan a cabo autoanálisis y autocríticas para seguir mejorando, cuestionan ideas tanto propias como de las personas que los rodean, se mantienen informados en las tendencias de los mercados laborales, tienen la habilidad de reaccionar con calma ante situaciones de adversidad y aprovechan al máximo los medios electrónicos.

Por su parte, los jóvenes del IPN, en comparación con los participantes del TecNM y los alumnos españoles, piensan que son capaces de mostrar flexibilidad cuando se presentan procesos de cambio, saben resolver conflictos, que generan nuevas ideas, sienten que pueden separar su esfera personal de lo laboral y piensan que los aprendizajes obtenidos han ayudado a fortalecer las actitudes de liderazgo.

Shakir (2009) enfatiza que en los programas de estudios se deben integrar las habilidades de comunicación, pensamiento crítico, habilidades para resolver problemas, trabajo en equipo, aprendizaje permanente, gestión de la información, habilidad emprendedora, ética y moral profesional y capacidad para liderar. Pool (2017) añade que 


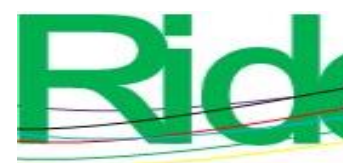

Revista Iberoamericana para la Investigación y el Desarrollo Educativo ISSN 2007 - 7467

se tiene que trabajar con el tema de la inteligencia emocional en las competencias blandas, ya que una persona que no es capaz de manejar sus emociones de forma efectiva puede tener consecuencias muy serias en relación con el trabajo en equipo.

Retomando lo extraído del análisis de la escuela del IPN, se halló que la carrera la ingeniería Industrial es la que mejor puntuó el factor de las habilidades blandas; por el contrario, la ingeniería en Informática es la que obtuvo la menor puntuación. Además, los jóvenes que indicaron que actualmente trabajan son los que mejor califican las habilidades blandas. Y los participantes que dijeron tener una beca son los que perciben mejor las habilidades. Por lo anterior, se está de acuerdo con Rao (2014) cuando enfatiza que es factible que se motive a los jóvenes para que tengan contacto con la industria, ya que el ambiente laboral es una plataforma que ayuda a trabajar sus talentos e incrementar las habilidades blandas. De igual forma, Musa, Mufti, Latiff y Amin (2012) señalan que los jóvenes deben ser capaces de identificar las habilidades blandas que están adquiriendo en el aula y deben tener la capacidad de trasladarlas al campo laboral, por lo que se debe fomentar las estancias en las empresas. Taylor (2016), finalmente, recomienda que los estudiantes trabajen en vacaciones en la industria, ya que esto permite que se desarrollen diversas competencias.

Ahora bien, el TecNM fue la institución, en comparación con las otras dos, cuyos jóvenes calificaron con más bajas puntuaciones la adquisición de competencias socioemocionales. Se halló que la carrera que mejor evalúa las habilidades blandas es la ingeniería Industrial; la que menos calificación otorgó es la ingeniería en Tecnologías de la Información y Comunicaciones. Igualmente, se encontró que los participantes que dijeron no tener asignaturas reprobadas son los que mejor perciben la adquisición de habilidades blandas.

Considerando los resultados obtenidos por las tres universidades, hay concordancia con lo que apuntan Majid et al. (2012), quienes enfatizan en la necesidad por parte de los jóvenes de concientizar sobre la importancia de las competencias blandas y las formas en cómo desarrollar y practicar tales. Aquí se recomienda que las universidades realicen talleres especializados que permitan que los jóvenes entrenen las competencias interpersonales. En esa misma línea, Direito, Pereira y de Oliveira (2012) anotan que los jóvenes deben hacerse responsables de su desarrollo profesional y, por lo tanto, deben trabajar en las deficiencias relacionadas con las competencias blandas, ya que no pueden estar dependiendo de los factores externos que estimulan la adquisición de estas. 


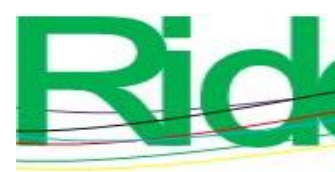

Revista Iberoamericana para la Investigación y el Desarrollo Educativo ISSN 2007 - 7467

Los resultados encontrados permiten visualizar un escenario complicado que requiere de trabajo e inversión en el rubro de capacitación por parte de las universidades. Los profesores son elementos sustanciales en el proceso de enseñanzaaprendizaje, ellos tienen el reto de transmitir conocimientos técnicos, pero también tienen que trabajar las habilidades socioemocionales de los estudiantes: demasiada responsabilidad para una infinidad de problemas a los que se enfrentan. Se tiene que trabajar en el bienestar de los profesores, por lo que es factible que se considere crear e implementar programas orientados a la inteligencia emocional de los académicos. Esto podría apoyar en entrenar a los académicos en las formas para evitar el estrés (Vesely y Saklofske, 2018; Dewaele, Gkonou y Mercer, 2018)

\section{Conclusiones}

Se concluye que las instituciones de educación superior tienen que redoblar esfuerzos para ayudar a los jóvenes a incrementar sus competencias socioemocionales. De acuerdo con lo expuesto y analizado, se recomienda estudiar las competencias socioemocionales que requieren los mercados laborales de los ingenieros para actualizar los programas de estudio y, de esta forma, tener egresados preparados para ingresar a un entorno plagado de competencia. En el caso del IPN, se le tiene que poner atención a los programas relacionados con la informática, ya que son los que obtuvieron menos puntos en el tema de las habilidades blandas.

Es imprescindible que las instituciones educativas trabajen con los jóvenes las competencias sociales que coadyuven a incrementar su liderazgo para que puedan aspirar a puestos estratégicos y directivos en las organizaciones. Sin embargo, debe quedar claro que, para alcanzar lo anterior, se requiere sobre todo de actitud, disposición e iniciativa por parte de los discentes. Por lo anterior, las escuelas tienen que reforzar los programas de estancias académicas con la industria, ya que los alumnos pueden experimentar los ambientes laborales y perder el posible miedo a resolver problemas y convivir con personas de mayor edad y experiencia. También se pueden trabajar con talleres que el alumnado puede cursar como asignaturas complementarias. 


\section{Referencias}

Abdullah-Al-Mamun, M. (2012). The soft skills education for the vocational graduate: Value as work readiness skills. British Journal of Education, Society \& Behavioral Science, 2(4), 326-338.

Bailly, F. and Léné, A. (2012). The personification of the service labour process and the rise of soft skills: a French case study. Employee Relations, 35(1), 79-97.

Balcar, J. (2014). Soft skills and their wage returns: Overview of empirical literature. Review of Economic Perspectives, 14(1), 3-15.

Berglund, A. and Heintz, F. (2014). Integrating Soft Skills into engineering education for increased student throughput and more professional engineers. Retrieved from https://www.lth.se/fileadmin/lth/genombrottet/konferens2014/11_Berglund_Hei ntz.pdf.

Cleary, J. L., Kerrigan, M. R. and Van Noy, M. (2017). Towards a New Understanding of Labor Market Alignment. In Paulsen, M. (ed.), Higher Education: Handbook of Theory and Research (pp. 577-629). Cham, Switzerland: Springer. Retrieved from https://doi.org/10.1007/978-3-319-48983-4_12.

Dell'Aquila, E., Marocco, D., Ponticorvo, M., di Ferdinando, A., Schembri, M. and Miglino, O. (2017). Educational Games for Soft-Skills Training in Digital Environments. Advances in Game-Based Learning. Cham, Switzerland: Springer.

Devis-Rozental C. (2018). Humanising Higher Education by Meeting Scholars' Needs with Socio-Emotional Intelligence. In Devis, C. (ed.), Developing SocioEmotional Intelligence in Higher Education Scholars. Cham, Switzerland: Palgrave Macmillan. Retrieved from https://doi.org/10.1007/978-3-31994036-6 5.

Dewaele, J. M., Gkonou, C. and Mercer S. (2018). Do ESL/EFL Teachers' Emotional Intelligence, Teaching Experience, Proficiency and Gender Affect Their Classroom Practice? In Martínez, J. (ed.), Emotions in Second Language Teaching. Cham, Switzerland: Springer. Retrieved from https://doi.org/10.1007/978-3-319-75438-3_8.

Direito, I., Pereira, A. and de Oliveira, A. M. (2012). Engineering undergraduates' perceptions of soft skills: Relations with self-efficacy and learning styles. Procedia-Social and Behavioral Sciences, 55, 843-851. Retrieved from sciencedirect.com/science/article/pii/S1877042812040335. 


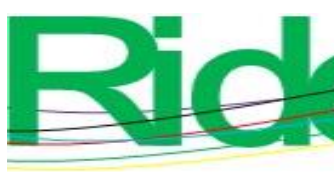

Revista Iberoamericana para la

Investigación y el Desarrollo Educativo

ISSN $2007-7467$

Fadel, C. and Groff J. S. (2019). Four-Dimensional Education for Sustainable Societies. In Cook, J. (ed.), Sustainability, Human Well-Being, and the Future of Education. Cham, Switzerland: Palgrave Macmillan. Retrieved from https://doi.org/10.1007/978-3-319-78580-6_8.

Florea, R. and Stray, V. (2018). Software Tester, We Want to Hire You! an Analysis of the Demand for Soft Skills. In Garbajosa, J., Wang, X. and Aguiar, A. (eds.), Agile Processes in Software Engineering and Extreme Programming. XP 2018. Cham, Switzerland: Springer.

Gibb, S. (2014). Soft skills assessment: theory development and the research agenda. International Journal of Lifelong Education, 33(4), 455-471.

Gilar, R., Pozo, T. y Castejón, J. L. (2018). Desarrollando la inteligencia emocional en educación superior: evaluación de la efectividad de un programa en tres países. Educación XX1, 22(1).

Ibarraran, P., Ripani, L., Taboada, B., Villa, J. M. and Garcia, B. (2014). Life skills, employability and training for disadvantaged youth: Evidence from a randomized evaluation design. IZA Journal of Labor \& Development, 3(1), 10.

Kumar, S. and Hsiao, J. K. (2007). Engineers learn "soft skills the hard way": Planting a seed of leadership in engineering classes. Leadership and Management in Engineering, 7(1), 18-23.

Lennox, A. and Roos, F. (2017). Preparing students for the workplace-development of soft skills. In Delener, N. and Schweikert, C. (eds.), Changing Business Environment: Gamechangers, Opportunities and Risks (pp. 354-360). United States: Global Business and Technology Association. Retrieved from researchgate.net/profile/Zhenning_Xu2/publication/320064694_MARKET_SEG MENTATION_USING_CLUSTER_ANALYSIS_AND_COMBINATORIAL_ FUSION/links/59cbb1e20f7e9bbfdc3b6bb2/MARKET-SEGMENTATIONUSING-CLUSTER-ANALYSIS-AND-COMBINATORIALFUSION.pdf\#page=373.

Majid, S., Liming, Z., Tong, S. and Raihana, S. (2012). Importance of soft skills for education and career success. International Journal for Cross-Disciplinary Subjects in Education, 2(2), 1037-1042.

Matteson, M. L., Anderson, L., \& Boyden, C. (2016). "Soft Skills": A Phrase in Search of Meaning. Portal: Libraries and the Academy, 16(1), 71-88. 


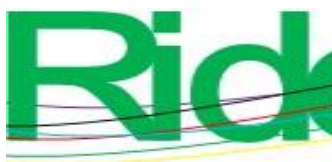

Revista Iberoamericana para la

Investigación y el Desarrollo Educativo

ISSN $2007-7467$

Musa, F., Mufti, N., Latiff, R. A. and Amin, M. M. (2012). Project-based learning (PjBL): inculcating soft skills in 21st century workplace. Procedia-Social and Behavioral Sciences, 59, 565-573.

Pieterse, V. and van Eekelen, M. (2016). Which Are Harder? Soft Skills or Hard Skills? In Gruner, S. (ed.), ICT Education. SACLA 2016 (pp 160-167). Cham, Switzerland: Springer. Retrieved from https://doi.org/10.1007/978-3-31947680-3_15.

Pool, L. D. (2017). Developing Graduate Employability: The CareerEDGE Model and the Importance of Emotional Intelligence. In Tomlinson, M. and Holmes, L. (eds.), Graduate Employability in Context. London, England: Palgrave Macmillan. Retrieved from https://doi.org/10.1057/978-1-137-57168-7_15.

Rao, M. S. (2014). Enhancing employability in engineering and management students through soft skills. Industrial and Commercial Training, 46(1), 42-48.

Retnanto, A., Parsaei, H. R. and Parsaei, B. (2019). Capacity Building Through Strengthening Professional Skills in Engineering Graduates. In Nazir, S., Teperi, A. M. and Polak-Sopińska, A. (eds), Advances in Human Factors in Training, Education, and Learning Sciences. Cham, Switzerland: Springer. Retrieved from https://doi.org/10.1007/978-3-319-93882-0_15.

Robles, M. M. (2012). Executive perceptions of the top 10 soft skills needed in today's workplace. Business Communication Quarterly, 75(4), 453-465. doi:10.1177/1080569912460400. Retrieved from https://doi.org/10.1177/1080569912460400.

Shakir, R. (2009). Soft skills at the Malaysian institutes of higher learning. Asia Pacific Education Review, 10(3), 309-315.

Taylor, E. (2016). Investigating the perception of stakeholders on soft skills development of students: Evidence from South Africa. Interdisciplinary Journal of e-Skills and Lifelong Learning, 12(1), 1-18.

Vesely A. K. and Saklofske D. H. (2018). Emotional Intelligence and the Next Generation of Teachers. In Keefer, K., Parker, J. and Saklofske, D. (eds.), Emotional Intelligence in Education. Cham, Switzerland: Springer. Retrieved from https://doi.org/10.1007/978-3-319-90633-1_14. 


\begin{tabular}{|c|c|}
\hline Rol de Contribución & Autor (es) \\
\hline Conceptualización & $\begin{array}{l}\text { Claudia Alejandra Hernández Herrera (igual) } \\
\text { Juan Carlos Neri Torres (igual) }\end{array}$ \\
\hline Metodología & Claudia Alejandra Hernández Herrera \\
\hline Software & $\begin{array}{l}\text { Claudia Alejandra Hernández Herrera (principal) } \\
\text { Juan Carlos Neri Torres (apoya) }\end{array}$ \\
\hline Validación & $\begin{array}{l}\text { Claudia Alejandra Hernández Herrera (principal) } \\
\text { Juan Carlos Neri Torres (apoya) }\end{array}$ \\
\hline Análisis Formal & Claudia Alejandra Hernández Herrera \\
\hline Investigación & $\begin{array}{l}\text { Claudia Alejandra Hernández Herrera (igual) } \\
\text { Juan Carlos Neri Torres (igual) }\end{array}$ \\
\hline Recursos & $\begin{array}{l}\text { Claudia Alejandra Hernández Herrera (principal) } \\
\text { Juan Carlos Neri Torres (apoya) }\end{array}$ \\
\hline Curación de datos & $\begin{array}{l}\text { Claudia Alejandra Hernández Herrera (principal) } \\
\text { Juan Carlos Neri Torres (apoya) }\end{array}$ \\
\hline $\begin{array}{l}\text { Escritura - Preparación del } \\
\text { borrador original }\end{array}$ & Claudia Alejandra Hernández Herrera \\
\hline $\begin{array}{l}\text { Escritura - Revisión y } \\
\text { edición }\end{array}$ & Claudia Alejandra Hernández Herrera \\
\hline Visualización & Claudia Alejandra Hernández Herrera \\
\hline Supervisión & Claudia Alejandra Hernández Herrera \\
\hline $\begin{array}{l}\text { Administración de } \\
\text { Proyectos }\end{array}$ & Claudia Alejandra Hernández Herrera \\
\hline Adquisición de fondos & Claudia Alejandra Hernández Herrera \\
\hline
\end{tabular}

